\title{
Factors determining semantic facilitation and interference in the cyclic naming paradigm
}

\author{
Eduardo Navarrete $^{1}$ *, Paul Del Prato ${ }^{2}$ and Bradford Z. Mahon ${ }^{2,3}$ * \\ 1 Dipartimento di Psicologia dello Sviluppo e della Socializzazione, University of Padova, Padova, Italy \\ ${ }^{2}$ Department of Brain and Cognitive Sciences, University of Rochester, Rochester, NY, USA \\ ${ }^{3}$ Department of Neurosurgery, University of Rochester Medical Center, Rochester, NY, USA
}

\section{Edited by:}

Albert Costa, University Pompeu

Fabra, Spain

Reviewed by:

David Howard, Newcastle University, UK

Gary Oppenheim, University of Illinois at Urbana-Champaign, USA

\section{*Correspondence:}

Eduardo Navarrete, Dipartimento di Psicologia dello Sviluppo e della

Socializzazione, University of Padova, Via Venezia 8, 35131 Padova, Italy. e-mail: enavarrete2007@gmail.com; Bradford Z. Mahon, University of Rochester, Meliora Hall, Rochester, NY 14627-0268, USA.

e-mail:mahon@rcbi.rochester.edu
The cyclic naming paradigm, in which participants are slower to name pictures blocked by semantic category than pictures in an unrelated context, offers a window into the dynamics of the mapping between lexical concepts and words. Here we provide evidence for the view that incremental adjustments to the connection weights from semantics to lexical items provides an elegant explanation of a range of observations within the cyclic naming paradigm. Our principal experimental manipulation is to vary the within-category semantic distance among items that must be named together in a block. In the first set of experiments we find that naming latencies are, if anything, faster for within-category semantically close blocks compared to within-category semantically far blocks, for the first presentation of items. This effect can be explained by the fact that there will be more spreading activation, and thus greater priming at the lexical level, for within-category semantically close blocks than within-category semantically far blocks. We test this explanation by inserting intervening filler items (geometric shapes), and show as predicted, that while intervening unrelated trials abolish short-lived semantic priming effects, the long-lag interference effect that is characteristic of this paradigm is unaffected. These data place new constraints on explanations of the cyclic naming effect, and related phenomena, within a model of language production.

Keywords: lexical access, speech production, semantic interference, semantic facilitation, semantic distance, cyclic naming paradigm

\section{INTRODUCTION}

Many object naming studies have explored lexical access in speech production by manipulating the semantic context within which speakers retrieve words from their mental lexicon. There are at least two motivations for that approach. The first is that lexical access in speech production is semantically driven. Thus, in the course of naming an object, speakers must access and select the semantic representation corresponding to the target name before initiating lexical access. The second motivation for manipulating the semantic context in which target pictures are named is because most extant theories of speech production model information flow between levels of processing in terms of spreading activation (e.g., Lupker, 1979; Dell, 1986; La Heij, 1988; Roelofs, 1992; Caramazza, 1997; Rapp and Goldrick, 2000; but see Bloem and La Heij, 2003). In the context of other assumptions about the dynamics of spreading activation (e.g., Dell, 1986; La Heij, 1988; Roelofs, 1992) current models assume that the amount of activation that one representation propagates to other linked representations is proportional to its level of activation. Thus, when naming a given picture (e.g., horse), semantically related concepts (e.g., ZEBRA, DOG, etc.) will become activated proportional to the degree to which they are semantically related to the target. In addition, most theoretical models also assume that the propagation between the semantic level and the lexical level follows the same principle. As a consequence, in the course of object naming, multiple lexical representations would be activated (i.e., the target word "horse" along with semantically related items, such as "zebra," and "dog").

According to the principle of spreading activation between semantic and lexical stages of processing, the prediction follows that any semantically related context should facilitate the semantic and lexical processing of target items. In line with this prediction, it is a well-established phenomenon in word recognition paradigms, such as lexical decision, that responses to target words are facilitated by semantically related primes (e.g., McRae and Boisvert, 1998). Such effects find a natural explanation in that the amount of activation that propagates from a prime to the target representation scales with the semantic similarity between the two words - the more similar they are, the more activation spreads to the target, and the faster will be the subsequent response to the target.

Semantic distance, or its inverse - semantic similarity describes the semantic overlap between two items. Thus, closely related items (e.g., HORSE-ZEBRA) share more semantic information than less close (or far) related items (HORSE-SHARK); this may be modeled either in terms of the amount of overlap of semantic features (e.g., Dell, 1986) or the nature and number of links that connect different concepts (e.g., Roelofs, 1992). According to the principle of spreading activation, as the semantic distance between two concepts decreases, more activation would propagate between them. Thus, one would predict that semantic 
effects on target processing (e.g., horse) would be greater when a within-category close item (zebra) has just been processed compared to when a within-category far item has just been processed (whale).

The same situation obtains in speech production experiments: when prime and target stimuli are presented on consecutive trials, a facilitation effect can emerge. For instance, Huttenlocher and Kubicek (1983) reported faster naming latencies in object naming when the object named on the preceding trial was semantically related than when it was not related (see also Sperber et al., 1979; Humphreys et al., 1988; Lupker, 1988; Biggs and Marmurek, 1990; Hartsuiker et al., 2005; and for similar results without naming the first object see Flores d'Arcais and Schreuder, 1987; Bajo, 1988). The semantic facilitation effects reported in those studies would be congruent with models of lexical access that assume that the time to select the target word is independent of the level of activation of non-target words; or specifically, the time required to select a word depends only on its own level of activation, and is not affected for instance, by the activation level of the last word that was named (Dell, 1986; Caramazza, 1997; Rapp and Goldrick, 2000, for similar conclusions in bilingual lexical access, see Finkbeiner et al., 2006).

However, semantic interference effects are also observed in production, and in fact, have received far more attention and been given far more theoretical prominence than facilitation effects. For instance, Wheeldon and Monsell (1994) required participants to name pictures and written definitions of objects and observed that naming latencies to picture targets (e.g., "shark") were slower when some trials previously a semantic coordinate word (e.g., "whale") was produced as a response to a written definition, compared to when a non-semantic coordinate was previously produced (e.g., "volcano"). Similar semantic costs are reported if instead of naming written definitions, participants name objects (Vitkovitch et al., 2006). Furthermore, when several objects of the same semantic category are presented to be named, the amount of interference is cumulative, so that the amount of the delay observed for each additional instance of the category that is named depends on the total number of exemplars of the same category that have already been named (Brown, 1981; Howard et al., 2006; Costa et al., 2009; Navarrete et al., 2010). Semantic interference, as observed in those studies, has generally been interpreted to indicate that lexical selection is a competitive process in which the time required to select the target word is affected by the levels of activation of non-target words (Roelofs, 1992; Levelt et al., 1999). It is not clear, however, if the theory of lexical selection by competition would (in and of itself) ever "predict" that semantic interference should be observed when different instances from the same category are separated by intervening trials.

Thus, one may derive the following broad empirical generalization: a semantic relationship between the target on trial $n$ and the picture named on trial $n-1$ leads to facilitation, while the same relationship that spans multiple trials leads to interference. Regarding the theoretical implications, semantic facilitation is consistent with models that assume that lexical selection depends on target activation only (that is, selection by activation models) but challenges models of lexical selection by competition; in contrast, semantic interference challenges selection by activation models and is consistent with competitive models.
Why do semantically related contexts lead to facilitation in recognition, facilitation in production with no intervening trials, and interference in production with intervening (unrelated) trials? As noted above, over the last two decades, the field has arrived at the view that interference effects in speech production are theoretically more informative than facilitation effects, in that interference (but not facilitation) effects inform a model of the dynamics of lexical selection (i.e., that it occurs by competition). The rationale in relation to the object naming studies described above is that the lexicalization process over an object (or definition) makes its corresponding lexical unit a stronger competitor when on a subsequent trial a semantically related object has to be named (Wheeldon and Monsell, 1994; Howard et al., 2006).

However, recent work indicates that the theory of lexical selection by competition is not without its problems. In particular, the theory must be reinforced against the range of semantic facilitation effects that have been observed, and which, prima facie, are contrary to its central prediction (for data and relevant discussions, see e.g., Roelofs, 1992, 2003; Caramazza and Costa, 2001; Damian et al., 2001; Bloem and La Heij, 2003; Costa et al., 2003; Costa et al., 2005; Bloem et al., 2004; Finkbeiner and Caramazza, 2006; Kuipers et al., 2006; Mahon et al., 2007). Even explanations of longlag semantic interference effects in picture naming require some additional maneuvering for the theory, such as that activation "lingers" at the lexical level for some time. But the fact that trial-totrial semantic relationships lead to facilitation and not interference indicates that, on that theory, activation cannot "linger" from the previous trial (see Howard et al., 2006). An alternative account of the inference effect proposes that there are incremental learning effects that are expressed as changes in the connection weights between semantic and lexical level representations. Thus, naming "horse" on a given trial will strengthen the connection between the concept HORSE and the lexical representation "horse" and, at the same time, weaken the connections between the concept and the lexical representations of semantic coordinates of the target (e.g., zebra, dog). On a subsequent trial on which "zebra" must be named, naming latencies will be slower because of the weaker connections (Oppenheim et al., 2010; see also Navarrete et al., 2010) ${ }^{1}$. The account in terms of incremental learning need not assume lexical selection by competition. An important issue, to which we return in the General Discussion, is how the incremental learning proposal of semantic interference, and more broadly the model of lexical selection in which it is embedded, can explain observations of semantic facilitation when there are no intervening filler items.

The main goal of the current research is to empirically characterize the boundaries of semantic interference and facilitation effects observed in the cyclic naming paradigm. In the standard version of this task participants are required to name a series of

\footnotetext{
"This is not to imply that there is one concept for the concepts "horse," "zebra," "whale," and so on - rather, on one way of modeling lexical semantics, concepts are decomposed into sets of features that are shared among items from the same category. Thus, strengthening and weakening effects occur because the same set of features is used to access one word, of they many words to which those features are connected. However, it is not unreasonable to imagine how such a mechanism could be implemented within models that do not assume features (e.g., Roelofs, 1992) and so we therefore remain agnostic on the representational structure of lexical semantics.
} 
pictures several times in two semantic contexts. In the homogenous semantic context, objects presented in a block belong to the same superordinate semantic category (e.g., horse, dog, cat). In the heterogeneous semantic context, objects in a block belong to different superordinate semantic categories (e.g., horse, table, lemon). Under those conditions, naming latencies in homogenous blocks are on average slower than in heterogeneous blocks - the socalled semantic blocking, or cyclic naming, effect (Damian et al., 2001; see Kroll and Stewart, 1994 for the first demonstration of the effect). Of particular interest is that in the cyclic naming paradigm, participants are required to name semantically related objects on consecutive trials, that is, without interleaved unrelated items between them. This situation raises the empirical question of whether the effects described above from sequential object naming tasks (semantic interference and semantic facilitation) interact with one another in cyclic naming tasks. The answer is yes. Within the cyclic naming paradigm, for the first presentation of items in a block, there is either no effect at all or there is a semantic facilitation effect characterized by faster object naming latencies in homogeneous blocks than in heterogeneous blocks (e.g., Belke et al., 2005a,b; Damian and Als, 2005; Abdel Rahman and Melinger, 2007). In the current research we explored the pattern of semantic effects in the cyclic naming task by manipulating the semantic distance among the items of the homogeneous blocks, and exploring this manipulation as a function of the factors cycle (i.e., repetition within a block) and lag (i.e., the number of intervening trials between a given trial and the previously named within-category item).

\section{THE CURRENT RESEARCH}

In the present set of experiments we set out to test the effect of manipulating within-category semantic distance on the pattern of facilitation and interference effects observed within the cyclic naming paradigm. According to the hypothesis of lexical selection by competition, all else equal, more interference is expected when pictures are named in blocks of within-category close items compared to when the same pictures are named in the context of within-category far items (see a similar prediction in Alario and Moscoso del Prado Martín, 2010). According to selection by activation accounts of lexical selection, all else equal, naming latencies should be faster for within-category close contexts compared to within-category far contexts. However, and of central importance, is that "all else may not be equal," in two ways.

1) First we will seek to understand how the effect of manipulating within-category semantic distance interacts with the critical factors of cycle and lag. As noted above, the cyclic naming effect appears only in later cycles (repetitions) and, more generally, intervening trials are necessary in order for interference effects to emerge. That is, the cost in the cyclic naming paradigm is not created by the immediately preceding trial in the block, but rather by the contextual effect created by the picture(s) processed several trials before. If anything, the immediately preceding picture in a homogenous block will yield facilitation and not interference. Thus, in testing for an effect of within-category semantic distance, we are really testing for what happens when two effects of contrasting polarity are directly pitted against one another - a long-lasting interference effect and a trial-by-trial facilitation effect. We will attempt to tease apart these two effects with correlational analyses, and by interspersing non-critical and unrelated filler trials.

2) The second reason why "all else may not be equal," is that according to the incremental learning account of interference effects (Oppenheim et al., 2010), the degree to which connections are strengthened or weakened is proportional to the strength with which lexical items are activated by semantics. Thus, manipulating semantic distance will affect the degree to which those connection weights are altered. This means, that even on a model that does not assume lexical selection by competition, the prediction can be made that a within-category semantically close context may lead to slower naming latencies than a within-category semantically far context. The difference between this prediction as extrapolated from the incremental learning hypothesis, and as made by lexical selection by competition, is that the incremental learning hypothesis but not the hypothesis of lexical selection by competition predicts the within-category interference effect will be observed only for long lags.

Damian and Als (2005) reported evidence that semantic facilitation in the cyclic naming paradigm is a short-lasting phenomenon while semantic interference is a long-lasting phenomenon. In Experiment 4a of their study, the semantic blocking effect was absent for the first occurrence of the items but emerged thereafter, remaining stable for the remainder of the presentations. In Experiment $4 \mathrm{~b}$ of their study, the same procedure as in Experiment 4a was used with the only difference that filler items from unrelated categories were dispersed throughout the blocks. According the authors, if the lack of the semantic interference for the first occurrence of the block was due to a mechanism of short-lasting semantic priming, such a mechanism should be attenuated when filler items are presented between the target items. Consistent with this, in Experiment $4 \mathrm{~b}$ the semantic blocking effect was observed in all presentations of the target items, and most notably, the first presentation (i.e., the first cycle).

We thus had three goals with the current set of experiments. The first goal was to characterize the effect of varying withincategory semantic contexts (within-category semantically close vs. within-category semantically far). This manipulation is important because it is a "pure" manipulation of semantic distance; unlike the contrast of homogenous vs. heterogeneous contexts, the manipulation of within-category semantic distance is not confounded with a manipulation of semantic category coordinate status. This allows us to test whether the incremental learning account can explain long-lag semantic interference. Two predictions can be outlined. If the mechanism underlying long-lasting semantic interference is incremental, then the prediction is made that within-category semantically close contexts will yield slower naming overall than within-category semantically far contexts. The reason why is that when the picture named on trial $n-2$ or $n-3$ was very close within-category (e.g., "zebra") to the picture on the current trial (e.g., "horse") then the connection between the semantic and lexical levels will be weaker than when the picture on a previous trial is relatively far within-category (e.g., 
whale) to the picture on the current trial ("horse"). If incremental learning is not the mechanism underlying long-lasting semantic interference, a model that does not assume lexical selection by competition predicts that within-category semantically close will be faster than within-category semantically far contexts, due to the presence of greater trial-to-trial priming (and no additional cost) created by the high semantic similarity among the pictures in a within-category semantically close block.

The second goal was to study the effect of intervening unrelated trials. Thus, in Experiments 1 and 2 there were no interleaved semantically unrelated filler items between the experimental items, while in Experiments 3a and 3b we included unrelated filler trials. The logic here is the same as in Damian and Als (2005), in that having interleaved unrelated trials should abolish short-lived semantic priming effects. By manipulating whether there are interleaved unrelated trials, we can directly study the emergence of a possible within-category semantic distance effect both "under the influence" of short-lived semantic priming and without short-lived semantic priming.

The third goal was to characterize the emergence of withincategory semantic distance effects as a function of the factor cycle (i.e., repetition within a block). One prediction made by the hypothesis that the cyclic naming effect (homogenous vs. heterogeneous) is due to incremental learning, is that (see Goal 1 above) within-category semantically close will be slower than withincategory semantic far. To anticipate the principal finding, that is the pattern that is obtained. Another prediction that is made is that the within-category semantic distance effect (close $>$ far) will not be present in the first cycle. That is because trials that occur later in a block will have many long-lag within-category items preceding them within the block, while trials early in the block will have fewer such items. Finally, models of lexical access agree on the assumption that more spreading activation is expected between semantically close related items than semantically far related items. Therefore, if semantic facilitation instead of interference is observed in the first cycle (replicating previous studies, e.g., Abdel Rahman and Melinger, 2007) an empirical question is whether the facilitation effect would depend on semantic distance among the items of the homogenous blocks.

In Experiment 1 semantic distance was manipulated between items. Homogenous blocks were created in such a way as to cover a large range of semantic distance. In Experiments 2 and 3 semantic distance was manipulated within items and the same items were presented in two homogenous blocks: within-category close items and within-category far items. Furthermore, in Experiment 3 a we choose pictures such that visual similarity among items in the within-category close condition was minimized so as to be comparable to the within-category far condition, while in Experiment $3 \mathrm{~b}$ we choose pictures such that visual similarity was allowed to be greater among the within-category semantically close pictures than among the within-category semantically far pictures.

\section{EXPERIMENT 1: MANIPULATING WITHIN-CATEGORY SEMANTIC DISTANCE BETWEEN ITEMS WITH NO INTERLEAVED UNRELATED TRIALS}

Eight semantic categories were selected for Experiment 1. Objects were presented in two semantic contexts. In the homogeneous context, objects within the blocks belonged to the same semantic category; in the heterogeneous context, objects came from different categories. Experiment 1 had several goals. First, we aimed to replicate the interaction between semantic context and position within a block, with facilitation or no effect for the first presentation, and then interference emerging over later presentations within the block (see Level I analysis below). Second, we aimed to explore whether the semantic effects (interference and/or facilitation) are modulated by the semantic distance among the items of the homogenous blocks. Semantic distance was determined by semantic similarity ratings among the items that appeared together in blocks. Specifically, after the main naming experiment participants were asked to judge the semantic similarity between all possible pairs of items that had been presented together in the homogeneous and the heterogeneous blocks. Finally, correlation analyses between the magnitude of the semantic context effect and the semantic ratings were carried out on an item-by-item basis and for each occurrence of the items within the blocks (see Level II analysis below).

One could argue that semantically related objects share more visual features than unrelated objects, and that this could affect the overall pattern of naming latencies in heterogeneous and homogenous blocks (e.g., Lotto et al., 1999). Two measures were taken in order to mitigate the influence of visual variables in the results. First, before starting the experiment proper participants were exposed three times to all of the experimental pictures (for a similar procedure see Abdel Rahman and Melinger, 2007). This massive pre-exposure was intended to mitigate the role of object identification variables during the main naming experiment. Second, visual similarity between homogeneous and heterogeneous blocks was determined and analyzed through a computational approach to measuring similarity in visual shape.

\section{METHOD \\ Participants}

Twelve native Italian speakers (students at the University of Trento, Italy) took part in the experiment. Participants in this and subsequent experiments had normal or corrected to normal vision and participated in only one experiment.

\section{Materials}

Sixty-four black and white photographs depicting objects from 8 semantic categories were selected. Photographs in this and subsequent experiments were taken from the Internet and sized to fit within a square of $400 \times 400$ pixels. See Section "Appendix A" for a list of materials.

\section{Design}

Each picture presented in two naming conditions. In the Homogeneous condition pictures were paired with three semantic coordinate pictures. In the Heterogeneous condition, pictures were paired with three pictures from different semantic categories. There were a total of 32 blocks with 4 pictures in each block. Half of the blocks belonged to the homogeneous naming condition and the other half to the heterogeneous naming condition. Within each block, each of the four pictures was presented four times in random order with the constraint that the same picture 
never appeared on consecutive trials. Thus, there were a total of four cycles within each block, corresponding to each of the four occurrences of the pictures. In this design a cycle would not necessarily coincide with four consecutive trials. Following Damian et al.'s (2001) design, the 32 experimental blocks were presented in a ABBA design: half of the participants started with 8 semantically related blocks followed by the 16 unrelated blocks and finished with the remaining 8 semantically related blocks; the other half of the participants started with 8 unrelated blocks, followed by the 16 semantically related blocks, and finished with the remaining unrelated blocks. There was a short pause of several seconds between each block.

Visual similarity among the items within homogeneous and heterogeneous conditions was assessed computationally. Each picture in each block was compared with the other three pictures of the same block. Similarity in visual shape was determined using the algorithm of Belongie et al. (2002), which computes the "cost" that would be required to warp an image into the shape of another image (see Mahon et al., 2007, for precedent on the use of this algorithm). By blocks, averages of visual similarity values for the items within the 16 homogenous blocks (mean $=0.132 \pm 0.03$ ) were not different than the averages of visual similarity values for the items within the 16 heterogeneous blocks ( mean $=0.129 \pm 0.03 ; t<1$ ).

\section{Procedure}

Participants were seated approximately $60 \mathrm{~cm}$ from the screen. The experimental session was divided into four parts. First, there was a familiarization phase in which each picture was presented with the corresponding name and participants were instructed to read the name while paying attention to the picture. Second, there was a training phase. The training consisted of 2 blocks containing the 64 pictures presented in a random order. During this phase participants were corrected by the experimenter when necessary. Third, was the experimental phase. In this phase each participant was presented with the 32 blocks and asked to name the pictures as fast and as accurately as possible. The fourth phase was a norming study in which participants were required to judge the semantic similarity between all possible pairs of items that had appeared together within the blocks.

Each trial within the experimental naming phase consisted of the following events. A fixation cross was shown in the center of the screen for $500 \mathrm{~ms}$ and was followed by a blank interval of $500 \mathrm{~ms}$. The picture was presented for $500 \mathrm{~ms}$. Response latencies were measured from the onset of the picture. The next trial started $1500 \mathrm{~ms}$ after the onset of participants' response or $3000 \mathrm{~ms}$ after the offset of the target. Stimulus presentation, response times, and response recording were controlled by the program DMDX (Forster and Forster, 2003).

For the semantic norming study words instead of pictures were used. Participants were presented with two words corresponding to pictures they had seen and were instructed to judge the semantic similarity between them (from $1=$ not related at all; to $7=$ very related). Each item was paired with all of the items with which it had appeared in a block (homogeneous and heterogeneous). Thus, each target word was presented a total of six times: three with semantically related items and three with semantically unrelated items. There were a total of 384 trials. The order of presentation of the trials and the position of the words (left or right) was random for each participant. The semantic rating study started after 20 practice trials using filler words.

\section{Analyses}

Four types of responses were excluded from the analyses of response times: (a) production of clearly erroneous picture names; (b) verbal disfluencies (stuttering, utterance repairs, and production of non-verbal sounds that triggered the voice key); (c) naming latencies less than $250 \mathrm{~ms}$ or greater than $1500 \mathrm{~ms}$; and (d) the first trial of each block. A total of $7.9 \%$ of the data points were excluded following those criteria.

Two different types of analysis were carried out. In the Level I analysis, two within-subject factors, Semantic Context (two levels: Homogenous and Heterogeneous) and Cycles within blocks (four levels: 1-4), and their interaction were modeled. In the Level II analysis we explored whether the semantic effects were affected by the semantic similarity between the items of the homogeneous blocks. We did that for each cycle and on an item-by-item basis. In order to avoid item-intrinsic properties affecting correlation values (as for instance, lexical frequency, or age of acquisition), the semantic blocking effect was calculated for each specific item by subtracting the latency in the heterogeneous block from the latency in the homogenous block. These differences, on an itemby-item basis, were then correlated with the semantic rating value of each item obtained in the related condition of the norming study (that is, the mean semantic rating obtained by comparing the semantic similarity of one specific item with all the other items of the homogenous block).

\section{RESULTS AND DISCUSSION}

Semantic ratings for homogenous and heterogeneous conditions were different (homogenous mean $=5.58 \pm 1.31$; heterogeneous mean $=1.62 \pm 0.97 ; t(30)=26.44, p<0.01)$.

\section{Level I analysis}

Separate analyses were carried out treating subjects and items as random factors, yielding $F 1$ and $F 2$ statistics, respectively. For all analyses in this article, degrees of freedom were GreenhouseGeisser corrected when the assumption of Sphericity was violated.

Mean naming latencies and error rates by condition are reported in Table $\mathbf{1}$ (see Figure 1). The analysis of naming latencies showed a main effect of Semantic Context $[F 1(1,11)=10.98$, $\left.p<0.01, \eta^{2}=0.54 ; F 2(1,63)=29.19, p<0.001, \eta^{2}=0.31\right]$, with slower response times for homogeneous blocks than for heterogeneous blocks. The main effect of Cycle was also significant $[F 1(3$, $33)=143.82, p<0.01, \eta^{2}=0.92 ; \quad F 2(1.83,115.59)=143.17$, $p<0.001, \eta^{2}=0.69$ ], with decreasing response times with each additional cycle. The interaction between Semantic Context and Cycle was significant $\left[F 1(3,33)=20.26, p<0.001, \eta^{2}=0.64\right.$; $\left.F 2(2.2,139.86)=18.89, p<0.01, \eta^{2}=0.23\right]$. This interaction reflects a polarity shift of the semantic effect between the first cycle and the remaining three. Paired $t$-tests revealed a semantic facilitation effect in the first cycle $[t 1(11)=-2.31 ; p<0.05$; $t 2(63)=-2.91 ; p<0.01]$ and semantic interference in the remaining cycles [Cycle $2: t 1(11)=4.29 ; p<0.01 ; t 2(63)=5.71$; $p<0.01$; Cycle $3: t 1(11)=5.25 ; p<0.01 ; t 2(63)=4.75 ; p<0.01$; Cycle $4: t 1(11)=3.31 ; p<0.01 ; t 2(63)=5.58 ; p<0.01]$. 
Table 1 | Mean naming latencies (RT), SDs in $\mathrm{ms}$, and percentage of error rates $(E)$ by semantic context and cycle in Experiment 1.

\begin{tabular}{llllllll}
\hline Cycle & \multicolumn{5}{c}{ Semantic context } \\
\cline { 2 - 5 } & \multicolumn{3}{c}{ Homogenous } & & \multicolumn{3}{c}{ Heterogeneous } \\
\cline { 2 - 3 } \cline { 6 - 8 } & RT & SD & E & & RT & SD & E \\
\hline 1 & 640 & 70 & 2.7 & & 655 & 66 & 2.5 \\
2 & 579 & 57 & 1.7 & & 548 & 61 & 1.8 \\
3 & 573 & 58 & 2.6 & & 544 & 61 & 1.3 \\
4 & 569 & 57 & 2 & & 542 & 66 & 0.9 \\
Mean & 587 & & 2.2 & 567 & & 1.6
\end{tabular}

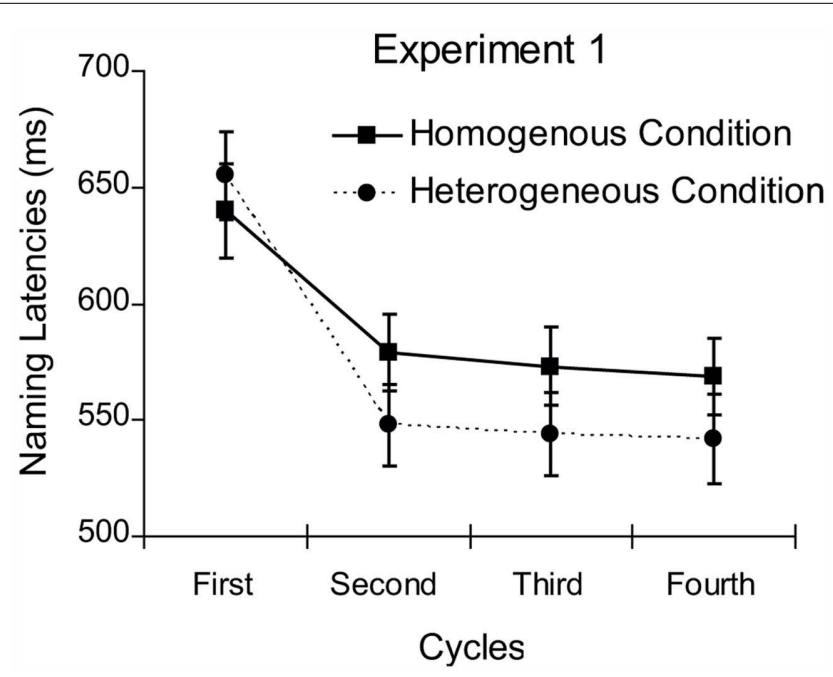

FIGURE 1 | Mean naming latencies by Semantic Context and Cycle for Experiment 1.

In the analysis of error rates the main effect of Semantic Context was not significant $\left[F 1(1,11)=1.9, p=0.19, \eta^{2}=0.14 ; F 2(1\right.$, $\left.63)=1.41, p=0.23, \eta^{2}=0.02\right]$, and the main effect of Cycle was significant only in the item analysis $[F 1(2.04,22.46)=1.8$, $\left.p=0.18, \eta^{2}=0.14 ; F 2(2.6,163.86)=4.42, p<0.01, \eta^{2}=0.06\right]$. The interaction between these two factors was not significant $(F \mathrm{~s}<1)$.

\section{Level II analysis}

The correlation between the semantic interference effect (RT homogenous-RT heterogeneous) and semantic ratings in the related condition was negative in Cycle $1(r=-0.27, p<0.04$, two tailed) and positive in the remaining cycles (Cycle 2: $r=0.25$, $p<0.05$; Cycle 3: $r=0.22, p=0.08$; Cycle 4: $r=0.39, p<0.01$; all two tailed).

Experiment 1 replicated the previous observation that in the first cycle, semantic facilitation is observed, with the interference effect emerging only for later cycles within the block. Of particular importance, however, is that variation in naming latencies as a function of within-category semantic distance followed the same pattern. For the first cycle, pictures in within-category semantically close contexts were named faster than pictures in within-category semantically far contexts. However, by the second cycle this effect completely reversed, in parallel to the emergence of the semantic blocking effect itself (i.e., related vs. unrelated). The design of Experiment 1 explored within-category semantic distance effects between items, as items appeared only once in a semantic homogenous block, and semantic distance was estimated across different homogenous blocks containing different items. In the next experiments we manipulated within-category semantic distance within items by presenting the same item in two homogenous blocks (one with within-category semantically close items and another with within-category semantically far items).

\section{EXPERIMENT 2: MANIPULATING WITHIN-CATEGORY SEMANTIC DISTANCE WITHIN ITEMS WITH NO INTERLEAVED UNRELATED TRIALS}

In this experiment the same pictures were presented in three different semantic contexts: within-category semantically close, within-category semantically far, and unrelated (or heterogeneous) blocks. In order to avoid strategic preparation of the response, item order within cycles was completely randomized and the duration of the fixation point was jittered. The same types of analyses as in Experiment 1 were carried out.

\section{METHOD}

Participants

Twelve native English speakers (students at the University of Rochester) took part in the experiment.

\section{Materials}

Fifty-four black and white photographs were selected. Items were organized in two groups containing three categories with nine items per category. Group 1 contained the categories animals, tools, and fruits/vegetables, and Group 2 contained the categories animals, tools, and vehicles. Each group was arranged in a $3 \times 3$ matrix, where each row contained a grouping of three highly semantic similar items (e.g., dog, wolf, fox) and formed the withincategory close condition, and each column contained three less similar items (e.g., dog, mouse, lizard) and formed the withincategory far condition. The heterogeneous condition was formed by selecting three items from different superordinate categories (e.g., dog, fork, orange). Phonological similarity was reduced or eliminated among all items that appeared together in a block (see Appendix B for all materials). Furthermore, pictures were chosen so as to eliminate, if possible, the tendency for withincategory semantically close groupings to be more visually similar than within-category semantically far groupings of items.

\section{DESIGN}

Pictures were grouped into blocks that were either within-category close (e.g., dog, wolf, and fox), within-category far (e.g. dog, mouse, and lizard), or heterogeneous (e.g. dog, fork, and orange). Items were repeated three times in each block, and blocks were therefore nine trials long. Item order within block was random. The first occurrence of each item was denoted as cycle 1, the second occurrence as cycle 2, and the last as cycle 3. As item order 
within the block was completely random, the same item could be presented on two consecutive trials. There were a total of 54 blocks, presented in a different random order for each participant. Participants completed the 486 trials in approximately $35 \mathrm{~min}$, with intermittent break periods between each block.

\section{Procedure}

The procedure was the same as in Experiment 1 with two differences: there was no training phase and the trial structure was different. On each trial a fixation cross was presented for either $160,240,320,400$, or $480 \mathrm{~ms}$. This jittering was introduced so that participants would not anticipate the timing of picture onset. Following the fixation cross was a blank period of $160 \mathrm{~ms}$ followed by the target picture. Targets were presented for $480 \mathrm{~ms}$. The offset of the target picture was followed by a blank screen for $992 \mathrm{~ms}$, during which time participants responded. There were a total of 486 trials. Participants completed a semantic distance rating study following completion of the main experiment. For the rating study, words instead of pictures were used: participants were presented with two words corresponding to pictures they had seen and were instructed to judge the semantic similarity between them (from $1=$ not related; to $7=$ very related). Each item was paired with all of the items with which it had appeared in a block (within-category semantically close, within-category semantically far, and unrelated). Thus, each target word was presented a total of six times: two with semantically close related items, two with within-category semantically far items, and two with semantically unrelated items. There were a total of 162 trials. The order of presentation of the trials was randomized for each participant.

\section{RESULTS AND DISCUSSION}

The mean semantic ratings between all three semantic contexts were different [using the averages for blocks: close vs. far: $t(34)=9.55, p<0.01$; close vs. unrelated: $t(34)=42.88, p<0.01$ : far vs. unrelated: $t(34)=15.61, p<0.01]$. The mean ratings were $6.0,4.3$, and 1.8 for close, far, and unrelated contexts, respectively (on a seven point scale).

\section{Analysis level I}

The same analyses as in Experiment 1 were performed. A total of $17.6 \%$ of the data points were excluded following the same criteria as were used in Experiment 1. In the analysis of naming latencies (see Table 2; Figure 2), the main effect of Semantic Context was not significant $(F s<1)$, while the main effect of Cycle was significant, $\left[F 1(2,22)=146.41, p<0.01, \eta^{2}=0.93\right.$; $\left.F 2(1.41,75.08)=219.7, p<0.01, \eta^{2}=0.8\right]$, with response times decreasing for each cycle. The interaction between Semantic Context and Cycle was significant $[F 1(4,44)=3.66, p<0.02$, $\left.\eta^{2}=0.25 ; F 2(3.12,165.39)=2.92, p<0.04, \eta^{2}=0.05\right]$. Paired samples $t$-tests between the within-category semantically close and the heterogeneous condition showed a semantic facilitation effect in the first cycle, with response times faster in the withincategory semantically close condition than in the heterogeneous condition $[t 1(11)=-2.5, p<0.03 ; t 2(53)=-2.01, p<0.05]$. No significant differences were observed in the second and third cycles [Cycle 2: $t 1(11)=1.7, p=0.1 ; t 2(53)=1.8, p=0.08$;
Table 2 | Mean naming latencies (RT), SDs in ms, and percentage of error rates (E) by semantic context and cycle in Experiment 2.

\begin{tabular}{|c|c|c|c|c|c|c|c|c|c|}
\hline \multirow[t]{3}{*}{ Cycle } & \multicolumn{9}{|c|}{ Semantic context } \\
\hline & \multicolumn{3}{|c|}{ Close related } & \multicolumn{3}{|c|}{ Far related } & \multicolumn{3}{|c|}{ Heterogeneous } \\
\hline & $\mathbf{R T}$ & SD & $E$ & $\mathbf{R T}$ & SD & E & $\mathbf{R T}$ & SD & $\mathbf{E}$ \\
\hline 1 & 668 & 48 & 11.3 & 676 & 57 & 12.7 & 689 & 56 & 9.7 \\
\hline 2 & 580 & 55 & 6.7 & 576 & 48 & 6 & 568 & 51 & 5.6 \\
\hline 3 & 581 & 45 & 7.4 & 582 & 52 & 6.4 & 572 & 50 & 4.4 \\
\hline Mean & 610 & & 8.5 & 611 & & 8.4 & 610 & & 6.6 \\
\hline
\end{tabular}

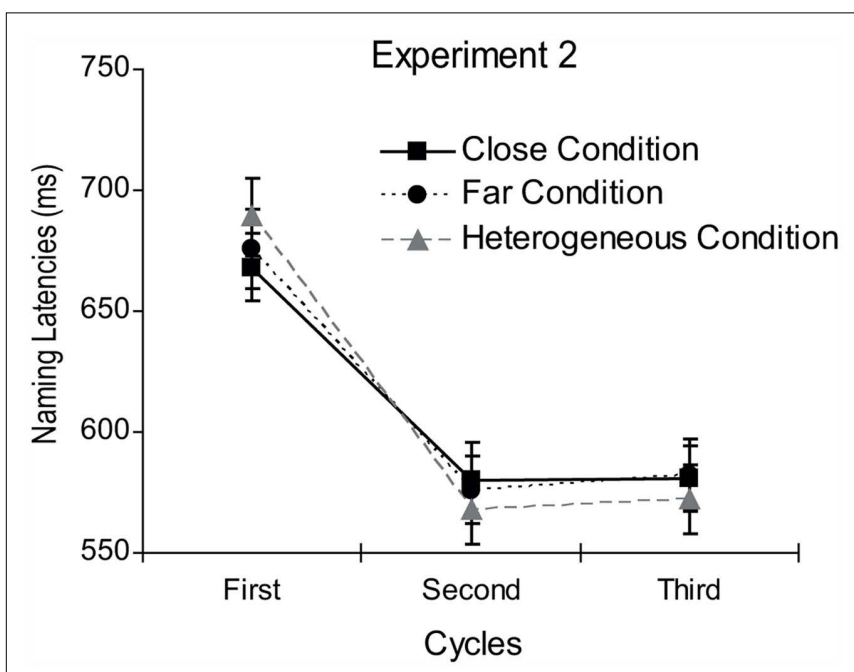

FIGURE 2 | Mean naming latencies by Semantic Context and Cycle for Experiment 2.

Cycle 3: $t 1(11)=1.4, p=0.18 ; t 2(53)=1.53, p=0.13]$. Paired samples $t$-tests between the within-category semantically far and the heterogeneous condition indicated no significant differences [Cycle 1: $t 1(11)=-1.51, p=0.15 ; t 2(53)=-1.33, p=0.18$; Cycle 2: $t 1(11)=1.18, p=0.26 ; t 2(53)=1, p=0.32$; Cycle 3 : $t 1(11)=1.59, p=0.13 ; t 2(53)=1.79, p=0.07]$. No significant differences were observed between the within-category semantically close and the within-category semantically far conditions $(t \mathrm{~s}<1)$.

There was no semantic interference effect comparing either the within-category semantically close or the within-category semantically far conditions to the unrelated baseline. In order to explore whether there was a semantic interference effect averaging over the two semantically related conditions, cycles 2 and 3 were collapsed, and related was compared to unrelated. A semantic blocking effect was obtained, with slower naming latencies in the semantically related condition than in the unrelated condition [580 and $570 \mathrm{~ms}$, respectively; marginally significant by subjects, $t 1(11)=1.98 ; p=0.07 ; t 2(53)=2.25 ; p<0.05]$.

In the analysis of error rates the main effect of Semantic Context was significant in the subject analysis $[F 1(2,2)=4.48, p<0.03$, 
$\left.\eta^{2}=0.29\right]$ and marginally significant in the item analysis [ $F 2(1.79$, 95.27) $\left.=2.72, p=0.07, \eta^{2}=0.04\right]$. The main effect of Cycle was not significant $\left[F 1<1 ; F 2(2,106)=1.62, p=0.2, \eta^{2}=0.03\right]$. The interaction between these two factors was not significant $(F s<1)$.

\section{Level II analysis}

The same analyses as in Experiment 1 were performed with the difference that now each item had two data points, one corresponding to the within-category semantically close vs. heterogeneous comparison and one corresponding to the within-category semantically far vs. heterogeneous comparison. Correlations were not significant $(p s>0.2)$.

As in Experiment 1, a semantic facilitation effect in the first cycle (for the within-category semantically close condition only) and an interaction between Semantic Context and Cycle were observed. In Experiment 2 no significant correlations were observed. The semantic blocking effect was observed only when collapsing cycles 2 and 3 together and collapsing the within-category close and far conditions together, and comparing related to unrelated.

\section{EXPERIMENT 3: MANIPULATING WITHIN-CATEGORY SEMANTIC DISTANCE WITHIN ITEMS WITH INTERLEAVED UNRELATED TRIALS}

Damian and Als (2005) reported that the interaction between semantic relationship and item repetition disappears when interleaved filler trials are presented in the first cycle of the block. According to those authors, this happens because the short-lasting semantic priming mechanism in the first cycle is attenuated by the presence of filler items. The same experimental blocks as in Experiment 2 were used in Experiment 3 with the difference that unrelated distractor objects (geometrical shapes) were intermixed between the target objects of the blocks. Following, Damian and Als' interpretation, both the semantic facilitation effect in the first cycle of the within-category close condition and the interaction between semantic context and item repetition found in Experiment 2, should disappear in Experiment 3. To ensure that any observed effects could not be explained by a concomitant manipulation of visual variables along with the manipulation of within-category semantic distance, two different versions of Experiment 3 were constructed. In Experiment 3 a we used the same photographs as in Experiment 2 (where visual similarity was minimized for within-category close blocks), and in Experiment $3 \mathrm{~b}$ we selected a new set of photographs of the same items with high visual similarity among the pictures in the within-category close condition. Previous research indicates that higher visual similarity among pictures will slow down response times (Lotto et al., 1999 , see also for a specific test in the cyclic paradigm, Belke et al., 2005b). Thus, it is important to be able to rule out, or at least reduce the possibility of, a contribution to a within-category distance effect of a confound of visual similarity with within-category semantic distance.

\section{METHOD}

\section{Participants}

Twenty-four native English speakers (students at the University of Rochester) took part in the experiment. Twelve participated in Experiment $3 \mathrm{a}$ and 12 in Experiment $3 \mathrm{~b}$.

\section{Materials}

The same experimental items used in Experiment 2 were used here. In order to create the filler condition, three unrelated objects (the geometrical shapes of a circle, a square, and a triangle) were selected and presented intermixed within the experimental objects in the blocks. Each experimental block contained 18 trials (nine objects and nine shape trials). The nine trials of objects and the nine trials of shapes corresponded to three repetitions (cycles) of presentation of the objects and shape images.

\section{Design and procedure}

The order of pictures and shapes within a block was random with the constraint that the first six trials contained the three object pictures and the three shapes, the second group of six trials contained the three objects and the three shapes, and the same for the final six trials. The first occurrence of the experimental items is denoted cycle 1 , the second cycle 2 , and the last cycle 3 . The same picture was never presented on adjacent trials. There were a total of 54 blocks, presented in a different random order for each participant. Participants completed the 972 trials in approximately $1 \mathrm{~h}$, with intermittent break periods between each block. The same procedure as in Experiment 2 was used here.

\section{RESULTS AND DISCUSSION}

Detailed analyses were performed by object and shape trials separately. We first present the analysis for the object trials. Following the same procedure as in previous experiments, $13.1 \%$ of the trials were removed from the analysis.

\section{Level I analysis}

In the analysis of naming latencies (see Table 3; Figure 3 ) there was a main effect of Semantic Context $[F 1(2,44)=20.61, p<0.01$, $\left.\eta^{2}=0.48 ; F 2(2,106)=35.14, p<0.01, \eta^{2}=0.39\right]$ and a main effect of Cycle $\left[F 1(1.47,32.51)=135.03, p<0.01, \eta^{2}=0.86\right.$; $\left.F 2(1.45,77.25)=214.74, p<0.01, \eta^{2}=0.8\right]$. The main effect of Experiment was significant $\left[F 1(1,22)=4.15, p=0.05, \eta^{2}=0.15\right.$; $\left.F 2(1,53)=152,4, p<0.01, \eta^{2}=0.74\right]$. The interaction between Semantic Context and Cycle was significant $[F 1(4,88)=5.48$, $\left.p<0.01, \eta^{2}=0.2 ; F 2(2.93,155.78)=6.21, p<0.01, \eta^{2}=0.1\right]$.

Table 3 | Mean naming latencies (RT), SDs in ms, and percentage of error rates $(E)$ by semantic context and cycle in Experiment $3 a$ and $3 b$.

\begin{tabular}{|c|c|c|c|c|c|c|c|c|c|}
\hline \multirow[t]{2}{*}{ Cycle } & \multicolumn{3}{|c|}{ Close related } & \multicolumn{3}{|c|}{ Far related } & \multicolumn{3}{|c|}{ Heterogeneous } \\
\hline & $\mathbf{R T}$ & SD & $\mathbf{E}$ & $\mathbf{R T}$ & SD & $\mathbf{E}$ & RT & SD & $\mathbf{E}$ \\
\hline \multicolumn{10}{|c|}{ EXPERIMENT 3A (VISUALLY DISSIMILAR ITEMS) } \\
\hline 1 & 698 & 60 & 15.3 & 690 & 53 & 14.4 & 682 & 55 & 16.4 \\
\hline 2 & 642 & 47 & 10.4 & 630 & 48 & 6.8 & 611 & 45 & 8.1 \\
\hline 3 & 644 & 57 & 10.3 & 620 & 43 & 7.7 & 601 & 41 & 6.3 \\
\hline Mean & 661 & & 12 & 647 & & 9.6 & 631 & & 10.3 \\
\hline \multicolumn{10}{|c|}{ EXPERIMENT 3B (VISUALLY SIMILAR ITEMS) } \\
\hline 1 & 757 & 80 & 11.9 & 756 & 72 & 10.6 & 753 & 79 & 12.8 \\
\hline 2 & 712 & 104 & 9.2 & 681 & 94 & 7 & 670 & 105 & 5.3 \\
\hline 3 & 699 & 93 & 7.6 & 674 & 110 & 7.3 & 661 & 109 & 5.9 \\
\hline Mean & 723 & & 9.6 & 704 & & 8.3 & 695 & & 8 \\
\hline
\end{tabular}



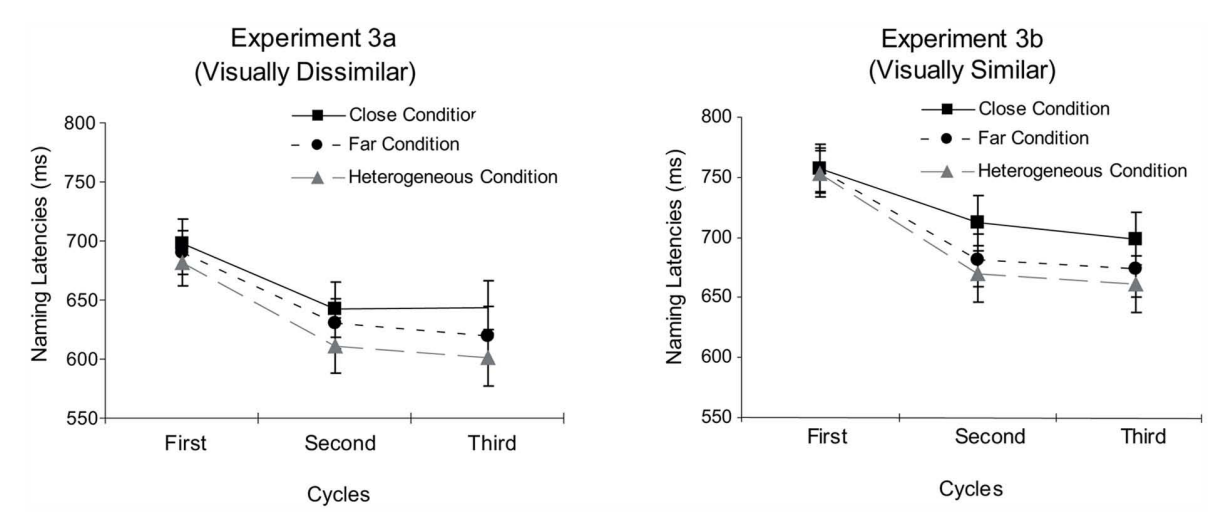

FIGURE 3 | Mean naming latencies by Semantic Context and Cycle for Experiments 3a and 3b.

No other interactions were significant $(p s>0.16)$. In order to provide a direct comparison with Experiment 2, the interaction between Semantic Context and Cycle was analyzed separately for Experiment 3a, confirming that for this Experiment the interaction was significant $\left[F 1(4,44)=2.84, p<0.04, \eta^{2}=0.2 ; F 2(3.08\right.$, 163.56) $\left.=3.35, p<0.02, \eta^{2}=0.06\right]$.

In order to further explore the interaction between Semantic Context and Cycle we conducted paired $t$-tests between all three semantic conditions. As there was no interaction between the factor Experiment and the other two factors, data points were collapsed across the factor Experiment. Comparisons between the semantically close and the heterogeneous conditions revealed no effect in the first cycle $[t 1(23)=1.64, p=0.11 ; t 2(53)=1.07$, $p=0.28]$, and semantic interference effects in the second and third cycles [Cycle 2: $t 1(23)=5.33, p<0.01 ; t 2(53)=7.09, p<0.01$; Cycle 3: $t 1(23)=5.95, p<0.01 ; t 2(53)=7.71, p<0.01]$. The same pattern was observed for the within-category semantically far condition: no effect in the first cycle $(t s<1)$, and semantic interference effects in cycles 2 and 3 [Cycle 2: $t 1(23)=2.95$, $p<0.01 ; t 2(53)=3.23, p<0.01$; Cycle $3: t 1(23)=4.66, p<0.01$; $t 2(53)=3.12, p<0.01]$. The comparison between the withincategory semantically close and far conditions was not significant in the first cycle $(t s<1)$ but significant in the last two cycles [Cycle 2: $t 1(23)=3.89, p<0.01 ; t 2(53)=4.94, p<0.01$; Cycle 3: $t 1(23)=3.9, p<0.01 ; t 2(53)=4.95, p<0.01]$, with slower response times in the within-category semantically close condition.

In the analysis of error rates the main effect of Semantic Context was significant $\left[F 1(2,44)=3.52, p<0.04, \eta^{2}=0.13 ; F 2(2\right.$, $\left.106)=5.59, p<0.01, \eta^{2}=0.09\right]$. The main effect of Cycle was also significant $\left[F 1(1.22,27.01)=279.27, p<0.01, \eta^{2}=0.92 ; F 2(1.41\right.$, $\left.75.05)=30.04, p<0.01, \eta^{2}=0.36\right]$. The main effect of Experiment was significant by items only $[F 1<1 ; F 2(1,53)=7.62$, $\left.p<0.01, \eta^{2}=0.12\right]$. The interaction between Semantic Context and Cycle was significant $\left[F 1(4,88)=2.87, p<0.03, \eta^{2}=0.16\right.$; $\left.F 2(4,212)=2.54, p<0.05, \eta^{2}=0.04\right]$. The interaction between Cycle and Experiment was significant in the item analysis only $\left[F 1<1 ; F 2(2,106)=4.23, p<0.02, \eta^{2}=0.07\right]$. The interaction between Semantic Context and Experiment was not significant $(F s>1)$.

\section{Analysis of shape trials}

As there were only three filler items (three geometrical shapes), analyses of the data from shape trials were performed only by Subjects. The analysis of naming latencies for shape trials showed no significant effects $(p s>0.18)$ for any of the factors. Mean latencies were $630 \mathrm{~ms}$ in the close condition, $632 \mathrm{~ms}$ in the far condition and $627 \mathrm{~ms}$ in the unrelated condition; and 627, 633, and $629 \mathrm{~ms}$ for the first, second, and third cycles, respectively. The analysis of error rates for shape trials also showed no significant effects (ps $>0.13)$.

\section{Level II analysis}

The same correlation analyses as in Experiment 2 were performed here. The correlation was not significant in the first Cycle $(p=0.7)$ and significantly positive in Cycles $2(r=0.42, p<0.01)$ and Cycle $3(r=0.38, p<0.01)$.

Several findings were reported in Experiment 3. First, a semantic blocking effect was reported; the presence of this effect is in contrast to Experiment 2 in which the same materials were used with the difference that there were no interleaved filler trials (geometrical shapes). Second, the magnitude of the semantic blocking effect was independent of the visual similarity of the pictures within the blocks, suggesting that the nature of the effect does not have a perceptual locus, consistent with previous work (e.g., Damian et al., 2001, but see Belke et al., 2005b, for effects of visual similarity in the same paradigm). Third, the semantic facilitation effect reported in the first cycle of the Close condition of Experiment 2 was absent in Experiment 3. The lack of the facilitation effect in the first cycle is congruent with Damian and Als (2005), suggesting that the presence of interleaved unrelated trials countermands the short-lasting facilitation effects. The final observation from Experiment 3 is that the effects were restricted to the object naming trials; the interleaved filler shape naming trials were unaffected by both context and cycle, replicating the pattern obtained by Damian and Als $(2005)^{2}$.

${ }^{2}$ However, contrary to the formal prediction of those authors, the interaction between the semantic blocking effect and cycle obtained in Experiment 2, without interleaved unrelated trials, was still present in Experiment 3. An explanation of this could be related to the differences between our design and that of Damian and 


\section{Analysis of lag}

In a further analysis we explored the influence of lag on the pattern of results. Even though this analysis is post hoc within the context of Experiment 3, it is of critical theoretical importance, since as discussed above, different theories make different predictions about short-lived facilitation vs. long-lived interference effects. Specifically, models that assume that lexical selection depends on target activation would predict semantic facilitation, while models that assume lexical selection by competition would predict semantic interference (see Introduction). In Experiment 3, cycles were six items long (three objects and three shapes). Thus, the minimum lag between two objects was 0 (i.e., two different objects presented on consecutive trials with no intervening filler trials) and the maximum lag could be 6 (i.e., three shape trials of cycle $n$, followed by the three shape trials of cycle $n+1$ separating the two different within-category items). As the presentation of the items within a block was pseudo-random, the total number of observations per each lag value varied. Specifically, the number of observations per Lag were: $\operatorname{lag} 0=3961, \operatorname{lag} 1=2955, \operatorname{lag} 2=1731$, $\operatorname{lag} 3=628$, lag $4=123$, lag $5=23$, and lag $6=16$ observations. In order to perform the analysis of the factor lag with a similar number of observations per cell, we collapsed lag values into three bins (lag 0 , $\operatorname{lag} 1$, and $\operatorname{lag} \geq 2$ ). Thus, the analysis had three factors (Semantic Context, Cycle, and Lag) with three levels each. Because any given item would not contribute equally to all levels of the Factor Lag for a given subject, item analyses are contraindicated and we therefore performed all analyses treating subjects as random factor.

Als (2005): (a) Damian and Als used eight items per block (four experimental, four filler) while we used only six (three experimental, three filler); (b) in their design, objects were presented under two semantic contexts (homogeneous and heterogeneous) while in our experiment there were three semantic contexts (close, far, and heterogeneous); (c) filler items in their study were from a pool of 16 pictures from diverse semantic categories while we choose 3 pictures corresponding to geometrical shapes.
In the analysis of naming latencies there were main effects of the three factors: $\operatorname{Lag}\left[F 1(2,46)=8.25, p<0.01, \eta^{2}=0.26\right]$, Semantic Context $\left[F 1(2,46)=17.16, p<0.01, \eta^{2}=0.42\right]$, and Cycle $\left[F 1(1.46,33.65)=162.66, p<0.01, \eta^{2}=0.87\right]$. The interaction between Lag and Semantic Condition $[F 1(4,92)=2.15$, $\left.p=0.08, \eta^{2}=0.08\right]$ was marginally significant. The interaction between Lag and Cycle was not significant $[F 1(2.49,57.3)=2.13$, $\left.p=0.11, \eta^{2}=0.08\right]$. Finally, the interaction between Semantic Condition and Cycle was (again) significant $[F 1(4,92)=3.63$, $p<0.01, \eta^{2}=0.13$; see Figure 4].

In the analysis of error rates, the main effect of Lag was not significant $\left[F 1(2,46)=1.62, p=0.2, \eta^{2}=0.06\right]$, the main effect of Semantic Context was marginally significant $[F 1(2,46)=3.06$, $\left.p=0.56, \eta^{2}=0.11\right]$, and the main effect of Cycle was significant $\left[F 1(1.17,27.12)=20.56, p<0.01, \eta^{2}=0.47\right]$. No interactions were significant $(p s>0.13)$.

\section{Modulation of repetition priming by the identity of an intervening trial}

In a final analysis, we studied the situation in which there was one intervening trial between repeats of the same picture. That intervening trial could be either an object or a shape. This presents an interesting situation, and allows further confirmation of our principal hypothesis. The general expectation is that repetition of a picture will lead to a decrease in response times. The question, however, is whether that decrease is modulated by context, and whether that contextual modulation is affected by whether the interleaved trial was an object or a shape. The results of this analysis are plotted in Figure 5. As can be seen, collapsing across the factor context, the overall amount of repetition priming is similar when the intervening trial is an object or a shape. However, and of particular interest, is that there is a clear polarity reversal along the dimension of within-category semantic distance according to the nature of the intervening stimulus. Specifically, when the intervening stimulus is a shape, the most repetition priming is observed

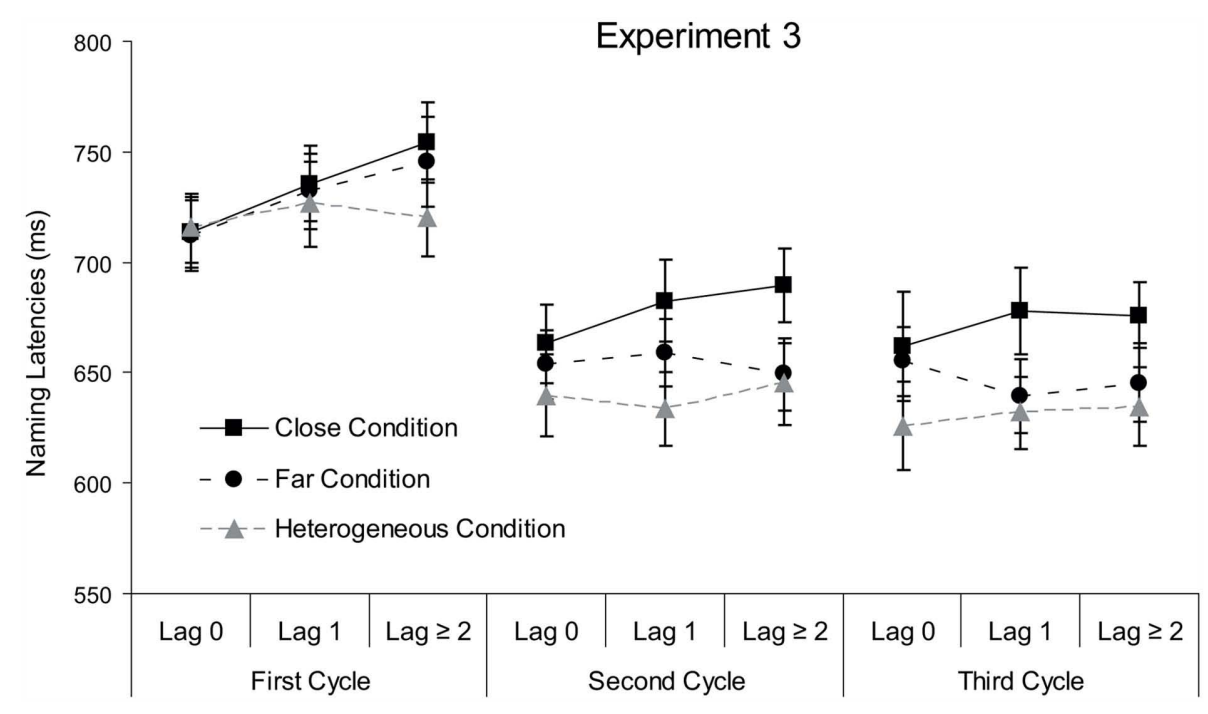

FIGURE 4 | Mean naming latencies by Semantic Context, Cycle, and Lag for Experiment 3. 


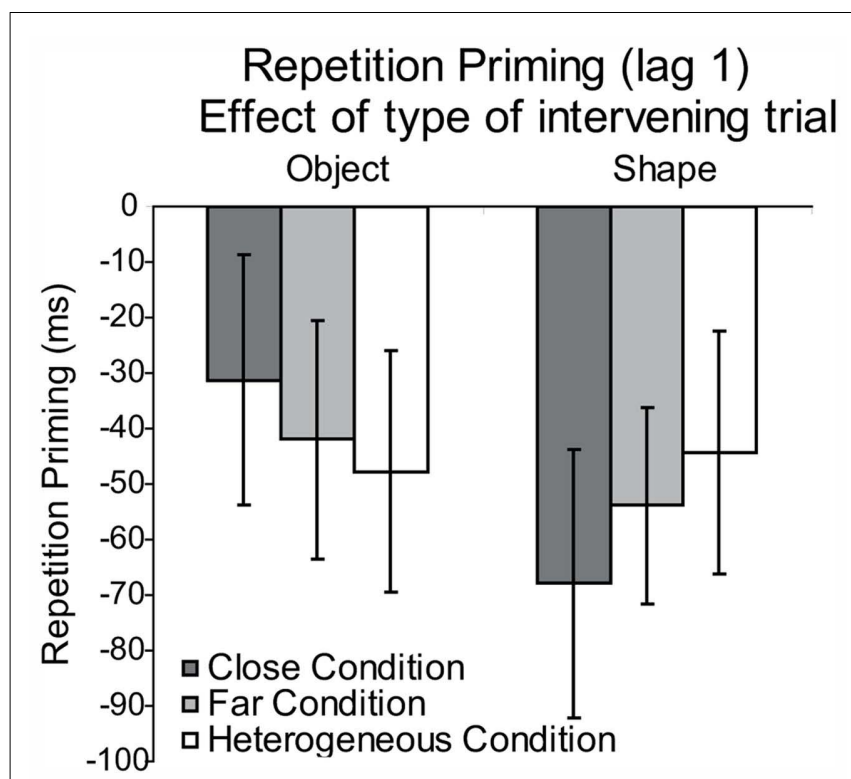

FIGURE 5 | Repetition Priming by Semantic Context and Cycle for Experiment 3 (in Lag 1).

for the within-category semantically close condition, followed by the within-category semantically far and finally the unrelated condition. This pattern is entirely inverted when the intervening trial was an object. These data, while inviting experiments specifically designed to test for these effects, strongly suggest that response times on a given trial in the cyclic naming paradigm are a composition of short-lived semantic facilitation and long-lag semantic interference.

\section{GENERAL DISCUSSION}

Several findings have been reported:

1) In the first cycle naming a picture is faster in a categorically related context (with no intervening unrelated trials) compared to an unrelated context. This was observed in the first cycle of Experiment 1 and in the first cycle of the within-category close condition of Experiment 2. This semantic facilitation replicates previous observations (e.g., Abdel Rahman and Melinger, 2007) and is comparable with other picture naming paradigms that report facilitation effects in object naming when the immediately preceding naming trial is categorically related (e.g., Huttenlocher and Kubicek, 1983). Importantly, our findings (Experiment 2) further show that this polarity reversal from facilitation to interference exists when semantic distance is manipulated within-category, as it does when comparing related to unrelated.

2) Further repetition of items within a block generates a semantic interference effect; that is, naming latencies are slower in semantically homogenous blocks than in heterogeneous blocks for later cycles. This is shown by the interference effects in cycles 2 through 4 of Experiment 1, and cycles 2 and 3 of Experiment 2 , replicating previous cyclic naming studies (e.g., Belke et al., 2005b).
3) The facilitation effect in the first cycle disappears when unrelated filler naming trials are embedded within the object naming trials, while the semantic interference effect that emerges for later cycles remains stable. This was observed comparing Experiments 2 and 3, and replicates Damian and Als' (2005) study.

4) Increasing the visual similarity among the items within a block slows down the overall naming latencies but does not influence the semantic effects (as reported for the comparisons between Experiments $3 \mathrm{a}$ and $3 \mathrm{~b}$ ); the first observation replicates previous studies on visual influences on object naming (Lotto et al., 1999), while the second observation suggests that the semantic effects in the cyclic naming task can be placed at a post-perceptual level of processing (Damian et al., 2001) ${ }^{3}$.

In summary, our results show that the way in which speaking is affected by semantic context depends on the joint influence of multiple factors. The theory of lexical selection by competition is not able to explain the fact that in the first cycle, a semantic facilitation effect (semantically related $<$ unrelated) as well as a facilitatory effect of within-category semantic distance (close $<$ far) is observed (Experiments 1 and 2). In this context, some authors have argued that facilitation effects suggest that lexical competition "needs some potentiation, through repetition of the items, to become observable" (Belke et al., 2005b, p. 687; for a similar argument see also, Abdel Rahman and Melinger, 2007); others argued that there is some type of a short-term semantic facilitation effect (Wheeldon and Monsell, 1994). But why would this be the case? Merely asserting this amounts to not much more than a redescription of the data.

Another approach is to pursue an explanation in terms of the incremental learning model outlined in the Introduction. Oppenheim et al. $(2007,2010)$ have proposed an interpretation of semantic interference effects in naming tasks that applies an errordriven learning mechanism. Naming an object would enhance the connectivity between the semantic and the lexical representations corresponding to the target picture, and, at the same time, it would weaken the connections to semantically related lexical units. These two aspects of the model can account, according to Oppenheim et al. (2010), for repetition priming effects and semantic interference effects in object naming tasks (for similar arguments see

\footnotetext{
${ }^{3}$ The differences between the within-category semantically close and withincategory semantically far conditions has been referred to as a "graded" effect. So-called graded semantic distance effects in the blocked naming task were investigated by Vigliocco et al. (2002). However, in that study semantic distance was manipulated between category and not within-category as we did here. For instance, target pictures (e.g., items from the category "clothing") were presented in blocks mixed with pictures belonging to a different category that was semantically close to the target category (e.g., "body parts") or semantically far from the target category (e.g., "vehicles"). Vigliocco and colleagues reported that naming latencies were on average slower in the former condition, the semantically close condition, than in the latter, the semantically far condition. In sum, our result would extend Vigliocco and colleagues' results to a within-category experimental design. The authors did not report analyses by the factor cycle (nor by lag). In order to provide a direct comparison with the study of Vigliocco et al. (2002), we conducted paired $t$-tests on naming latencies between the close and far conditions of Experiment 3 collapsing across cycle. The analysis showed slower naming latencies in the close condition than in the far condition $[t 1(23)=4.24, p<0.01 ; t 2(53)=5.24, p<0.01]$, converging with Vigliocco and colleagues' findings but using a within-category distance design.
} 
Vitkovitch and Humphreys, 1991; Navarrete et al., 2010). However, it is still unclear whether such a model can account for the semantic facilitation effect as a consequence of having named a semantically related picture in the immediately preceding trial. One possibility is that it takes "some time" for the weakening mechanism to operate. That is, while the first mechanism of strengthening of the semantic-to-lexical connections over the target picture (yielding repetition priming) is immediate, the inhibitory mechanism of weakening the connections between semantic and lexical related words would appear later. In other words, just as for lexical selection by competition, some type of post hoc mechanism would have to be envisioned that would effectively prevent incremental learning from occurring on a trial-to-trial basis. If such a mechanism were postulated, then because the incremental learning account is articulated within a theory of lexical selection in which the most highly activated word is selected regardless of the activation levels of non-target words, then the trial-to-trial facilitation would be explained. But then we are left, again, with the question of: Why? - Why does incremental learning not occur on a trial-bytrial basis? This could be an avenue to pursue but at least in the present context, such an account would be no less post hoc than the claim that "lexical selection requires potentiation through repetition." Both accounts amount to either redescriptions of the data or post hoc and unsupported assertions that deal simply with this particular fact.

Another route to resolving these issues may be suggested by the data reported in Figure 5. Perhaps the cyclic naming effect has to be understood as a modulatory effect of repetition priming. What appears to be a "semantic interference effect" is really reduced repetition priming in the more related condition compared to the less related condition. For the first presentation of items there is, if anything, semantic facilitation - both comparing related to unrelated, and within-category close to within-category far. That effect (by hypothesis) is what tells us about lexical selection, as the RT effects for the first presentation have been "contaminated" by neither repetition nor by long-lasting interference effects. How does one get the interference effect from repetition priming? From the initial baseline that is established by the first presentation of the items, all response times speed up with repetition; the amount that latencies speed up with repetition, however, will be affected (by hypothesis) by the strength of the connection that obtains from semantics to their corresponding lexical items. For pictures that appear in the context of semantically more similar items, their semantic-to-lexical connections will be relatively weaker than pictures that appear in the context of semantically more distant items. This is because the degree to which incremental learning weakens non-target semantic-to-lexical representations would be affected by the semantic similarity between the target and non-target words. This type of an explanation would be able to explain the full pattern of findings, including: (1) the observation of initial semantic facilitation (lexical selection is not by competition), (2) the emergence of semantic interference with repetition ("semantic interference" is really less repetition priming), (3) the observation that by the last cycle within-category, semantically close is slower than within-category semantically far (incremental learning is modulated by semantic distance), and (4) having unrelated filler items abolishes semantic facilitation effects in the first cycle but does not affect the emergence of the "semantic interference effect" (the interference effect is caused by differential weakening of semantic-to-lexical connections, and hence is a long-lag effect, while semantic facilitation is a trial-to-trial priming effect).

This type of an explanation is able to explain findings that are otherwise difficult to explain in terms of lexical competition. For instance, the hypothesis of lexical competition would have difficulty explaining semantic interference effects that can be induced merely by a change of instruction - instructions cannot change or modulate structural properties of the system, and lexical competition is, by hypothesis, a structural property of the system. However, instructions can affect the way in which different concepts are conceived as being related to one another, and so could reasonably affect the dynamics of which connections are incrementally weakened. In line with this theoretical approach, Abdel Rahman and Melinger (2011) recently reported that semantic interference in the cyclic naming task can be modulated by providing information to the participants about the relation of the items of the blocks. Besides the homogenous and heterogeneous conditions, Abdel Rahman and Melinger included a condition in which the items come from different semantic categories but belong to a common event or theme. For instance, "coffee," "knife," "stool," "bucket," and "creek" are not categorically related items but they can be potentially integrated into the common theme of a "fishing trip." Two versions of the cyclic naming task were constructed. In one version participants performed the standard task and, while the semantic blocking effect emerged, there was no difference between the thematic and the heterogeneous conditions. Interestingly, in a second version, participants were provided with a title before initiating each block that served to provide a theme for grouping or relating the items within the blocks. For instance, the title "foods" for the homogenous block containing the food items, the title "fishing trip" for the thematic block containing the items ("coffee," "knife," "stool," "bucket," and "creek") and the title "clothing" for a heterogeneous block without clothes items. Under these conditions, a thematic interference effect was reported: naming latencies in the thematic blocks were slower than in the heterogeneous blocks. According to Abdel Rahman and Melinger, "these findings suggest that semantic activation spread during speech planning can be modulated and flexibly adapted as a given context or situation dictates" (page 157; for similar arguments, see Experiment 3 in Wheeldon and Monsell, 1994). Our suggestion here is that the incremental learning model developed by Oppenheim et al. (2010) presents a more "flexible" characterization of the dynamics of lexical access during speech production than the hypothesis of lexical selection by competition.

A second finding relevant to the dynamics of flexibility in speech production that can be explained straightforwardly by the incremental learning model was reported by Belke et al. (2005b). In Experiment 3 of that study, Belke and colleagues studied whether there is transfer of the semantic blocking effect, as observed for repeated items, to new (previously unnamed) items. In that experiment, there could be either eight or four cycles of a given item within a block. Specifically, for half of the blocks the same items were presented across the eight cycles, 
while for the other half of the blocks cycles $5-8$ contained different semantic-category exemplars as the items presented in cycles 1-4. Belke and colleagues found that the magnitude of the semantic effect was not modulated according to whether or not the items were old or new in the last four cycles of the block. These findings suggest that the semantic effect generalizes to new, previously unnamed items. This generalization to new, unnamed items was interpreted by Belke and colleagues as evidence that it takes some time to potentiate the lexical competition mechanism in a cyclic paradigm. The finding is also consistent with the view, however, that the semantic effect reflects weakening of semantic-to-lexical connections for non-target items.

\section{REFERENCES}

Abdel Rahman, R., and Melinger, A. (2007). When bees hamper the production of honey: lexical interference from associates in speech production. J. Exp. Psychol. Learn. Mem. Cogn. 33, 604-614.

Abdel Rahman, R., and Melinger, A. (2011). The dynamic microstructure of speech production: semantic interference built on the fly. $J$. Exp. Psychol. Learn. Mem. Cogn. 37, 149-161.

Alario, F.-X., and Moscoso del Prado Martín, F. (2010). On the origin of the cumulative semantic inhibition effect. Mem. Cognit. 38, 57-66.

Bajo, M. T. (1988). Semantic facilitation with pictures and words. $J$. Exp. Psychol. Learn. Mem. Cogn. 14, 579-589.

Belke, E., Brysbaert, M., Meyer, A. S., and Ghyselinck, M. (2005a). Age of acquisition effects in picture naming: evidence for a lexical-semantic competition hypothesis. Cognition 96, B45-B54.

Belke, E., Meyer, A. S., and Damian, M. F. (2005b). Refractory effects in picture naming as assessed in a semantic blocking paradigm. Q. J. Exp. Psychol. 58A, 667-692.

Belongie, S., Malik, J., and Puzicha, J. (2002). Shape matching and object recognition using shape contexts. IEEE Trans. Pattern Anal. Mach. Intell. 24, 509-522.

Biggs, T., and Marmurek, H. (1990). Picture and word naming: is facilitation due to processing overlap? Am. J. Psychol. 103, 81-100.

Bloem, I., and La Heij, W. (2003). Semantic facilitation and semantic interference in word translation: implications for models of lexical access. J. Mem. Lang. 48, 468-488.

Bloem, I., van den Boogaard, S., and La Heij, W. (2004). Semantic facilitation and semantic interference in language production: further evidence for the conceptual selection model of lexical access. J. Mem. Lang. 51, 307-323.

Brown, A. S. (1981). Inhibition in cued retrieval. J. Exp. Psychol. Hum. Learn. 7, 204-215.

Caramazza, A. (1997). How many levels of processing are there in lexical access? Cogn. Neuropsychol. 14, 177-208.

Caramazza, A., and Costa, A. (2001). Set size and repetition in the pictureword interference paradigm: implications for models of naming. Cognition 80, 291-298.

Costa, A., Alario, F.-X., and Caramazza, A. (2005). On the categorical nature of the semantic interference effect in the picture-word interference paradigm. Psychon. Bull. Rev. 12, 125-131.

Costa, A., Mahon, B., Savova, V., and Caramazza, A. (2003). Level of categorization effect: a novel effect in the picture-word interference paradigm. Lang. Cogn. Processes 18, 205-233.

Costa, A., Strijkers, K., Martin, C., and Thierry, G. (2009). The time-course of word retrieval revealed by eventrelated brain potentials during overt speech. Proc. Natl. Acad. Sci. U.S.A. 106, 21442-21446.

Damian, M. F., and Als, L. C. (2005). Long-lasting semantic context effects in the spoken production of object names. J. Exp. Psychol. Learn. Mem. Cogn. 31, 1372-1384.

Damian, M. F., Vigliocco, G., and Levelt, W. J. M. (2001). Effects of semantic context in the naming of pictures and words. Cognition 81, B77-B86.

Dell, G. S. (1986). A spreadingactivation theory of retrieval in sentence production. Psychol. Rev. 3, 283-321.

Finkbeiner, M., and Caramazza, A. (2006). Now you see it, now you don't: on turning semantic interference into facilitation in a Stroop-like task. Cortex 42, 790-796.

In summary, then, our findings indicate that models of the cyclic naming effect based on lexical competition have difficulty explaining the presence of semantic facilitation in the first cycle when there are no interleaved unrelated filler items. We have argued that the incremental learning model of Oppenheim et al. (2010) is able to accommodate the "emergence" of the semantic interference effect in later cycles, if it is assumed that the interference reflects reduced repetition priming. This hypothesis can be directly evaluated with future work. The cyclic naming paradigm is fertile ground for studying the dynamics of word retrieval in speech production and will undoubtedly continue to serve as an important venue for adjudicating among different models of lexical selection.

Finkbeiner, M., Gollan, T., and Caramazza, A. (2006). Bilingual lexical access: what's the (hard) problem? Biling. (Camb. Engl.) 9, 153-166.

Flores d'Arcais, G. B., and Schreuder, R. (1987). Semantic activation during object naming. Psychol. Res. 49, 153-159.

Forster, K. I., and Forster, J. C. (2003). DMDX: a windows display program with millisecond accuracy. Behav. Res. Methods Instrum. Comput. 35, 116-124.

Hartsuiker, R. J., Pickering, M. J., and de Jong, N. H. (2005). Semantic and phonological context effects in speech error repair. J. Exp. Psychol. Learn. Mem. Cogn. 5, 921-932.

Howard, D., Nickels, L., Coltheart, M. and Cole-Virtue, J. (2006). Cumulative semantic inhibition in picture naming: experimental and computational studies. Cognition 100, 464-482.

Humphreys, G. W., Riddoch, M. J., and Quinlan, P. T. (1988). Cascade processes in picture identification. Cogn. Neuropsychol. 5, 67-103.

Huttenlocher, J., and Kubicek, L. F. (1983). The source of relatedness effects on naming latency. $J$. Exp. Psychol. Learn. Mem. Cogn. 9, 486-496.

Kroll, J. F., and Stewart, E. (1994) Category interference in translation and picture naming: evidence for asymmetric connections between bilingual memory representations. $J$. Mem. Lang. 33, 149-174.

Kuipers, J. R., La Heij, W., and Costa, A. (2006). A further look at semantic context effects in language production: the role of response congruency. Lang. Cogn. Process. 21, 892-919.

La Heij, W. (1988). Components of Stroop-like interference in picture naming. Mem. Cognit. 16, 400-410.

Levelt, W. J. M., Roelofs, A., and Meyer, A. S. (1999). A theory of lexical access in speech production. Behav. Brain Sci. 22, 1-75.

Lotto, L., Job, R., and Rumiati, R. (1999). Visual effect in picture and word categorization. Mem. Cognit. 27, 674-684.

Lupker, S. J. (1979). The semantic nature of response competition in the picture-word interference task. Mem. Cognit. 7, 485-495.

Lupker, S. J. (1988). Picture naming: an investigation of the nature of categorical priming. J. Exp. Psychol. Learn. Mem. Cogn. 14, 444-455.

Mahon, B. Z., Milleville, S. C., Negri, G. A., Rumiati, R. I., Caramazza A., and Martin, A. (2007). Actionrelated properties shape object representations in the ventral stream. Neuron 55, 507-520.

McRae, K., and Boisvert, S. (1998). Automatic semantic similarity priming. J. Exp. Psychol. Learn. Mem. Cogn. 24, 558-572.

Navarrete, E., Mahon, B. Z., and Caramazza, A. (2010). The cumulative semantic cost does not reflect lexical selection by competition. Acta Psychol. (Amst.) 134, 279-289.

Oppenheim, G. M., Dell, G. S., and Schwartz, M. F. (2007). Cumulative semantic interference as learning. Brain Lang. 103, 175-176.

Oppenheim, G. M., Dell, G. S., and Schwartz, M. F. (2010). The dark side of incremental learning: a model of cumulative semantic interference during lexical access in speech production. Cognition 114, 227-252.

Rapp, B., and Goldrick, M. (2000). Discreteness and interactivity in spoken word production. Psychol. Rev. 107, 460-499.

Roelofs, A. (1992). A spreadingactivation theory of lemma retrieval in speaking. Cognition 42, 107-142.

Roelofs, A. (2003). Goal-referenced selection of verbal action: modeling attentional control in the Stroop task. Psychol. Rev. 110, 88-125. 
Sperber, R. D., McCauley, C., Ragain, R. D., and Weil, C. M. (1979). Semantic priming effects on picture and word processing. Mem. Cognit. 7, 339-345.

Vigliocco, G., Vinson, D. P., Damian, M. F., and Levelt, W. J. M. (2002). Semantic distance effects on object and action naming. Cognition 85, B61-B69.

Vitkovitch, M., Cooper-Pye, E., and Leadbetter, A. (2006). Semantic priming over unrelated trials: evidence for different effects in word and picture naming. Mem. Cognit. 34, 715-725.

Vitkovitch, M., and Humphreys, G. W. (1991). Perseverant naming errors in speeded picture naming: its in the links. J. Exp. Psychol. Learn. Mem. Cogn. 17, 664-680.

Wheeldon, L. R., and Monsell, S. (1994). Inhibition of spoken word production by priming a semantic competitor. J. Mem. Lang. 33, 332-356.
Conflict of Interest Statement: The authors declare that the research was conducted in the absence of any commercial or financial relationships that could be construed as a potential conflict of interest.

Received: 07 August 2011; accepted: 02 February 2012; published online: $21 \mathrm{Feb}$ ruary 2012.

Citation: Navarrete E, Del Prato $P$ and Mahon BZ (2012) Factors determining semantic facilitation and interference in the cyclic naming paradigm. Front. Psychology 3:38. doi: 10.3389/fpsyg.2012.00038

This article was submitted to Frontiers in Language Sciences, a specialty of Frontiers in Psychology.

Copyright (๑) 2012 Navarrete, Del Prato and Mahon. This is an open-access article distributed under the terms of the Creative Commons Attribution Non Commercial License, which permits noncommercial use, distribution, and reproduction in other forums, provided the original authors and source are credited. 


\section{APPENDICES \\ APPENDIX A}

Materials used in Experiment 1 organized by Homogenous and Heterogeneous blocks. Italian translations are provided after the English name.

\section{Homogenous blocks}

Donkey (asino), horse (cavallo), pig (maiale), pecora (sheep)

Submarine (sottomarino), airplane (aereo), bicycle (bicicletta), train (treno)

Bed (letto), hammock (amaca), bench (panchina), chair (sedia)

Cabinet (armadio), drawer (cassetiera), sofa (divano), table (tavolo)

Coconut (cocco), banana (banana), pear (pera), grape (uva)

Helicopter (elicottero), motorcycle (motocicletta), tractor (trattore), ship (nave)

Bottle (bottiglia), cup (tazza), chalice (calice), flask (fiasco)

Shirt (camicia), skirt (gonna), sweater (maglione), dress (vestito)

Car (macchina), truck (camion), van (furgone), bus (pullman)

Hat (cappello), glove (guanto), scarf (sciarpa), belt (cintura)

Spider (ragno), goat (capra), owl (gufo), penguin (pinguino)

Drum (tamburo), guitar (chitarra), piano (pianoforte), saxo-

phone (sassofono)

Swan (cigno), cat (gatto), elephant (elefante), lizard (lucertola)

Alligator (coccodrillo), turtle (tartaruga), frog (rana), snake (serpente)

Scoop (paletta), fork (forchetta), spoon (cucchiaio), ladle (mestolo)

Record player (giradischi), radio (radio), stereo (stereo), television (televisione)

\section{Heterogeneous blocks}

Goat (capra), car (macchina), drum (tamburo), dress (vestito) Cabinet (armadio), chalice (calice), elephant (elefante), train (treno)

Banana (banana), helicopter (elicottero), frog (rana), stereo (stereo)

Bicycle (bicicletta), horse (cavallo), glove (guanto), ladle (mestolo)

Fork (forchetta), cat (gatto), bus (pullman), scarf (sciarpa)

Coconut (cocco), lizard (lucertola), bench (panchina), submarine (sottomarino)

Ship (nave), guitar (chitarra), sofa (divano), pig (maiale)

Alligator (coccodrillo), skirt (gonna), motorcycle (motocicletta), pear (pera),

Drawer (cassetiera), belt (cintura), penguin (pinguino), television (televisione)
Bottle (bottiglia), shirt (camicia), turtle (tartaruga), piano (pianoforte)

Truck (camion), flask (fiasco), spider (ragno), table (tavolo)

Spoon (cucchiaio), saxophone (sassofono), pecora (sheep), grape (uva)

Airplane (aereo), hat (cappello), record player (giradischi), snake (serpente)

Owl (gufo), bed (letto), sweater (maglione), cup (tazza), Donkey (asino), van (furgone), radio (radio), chair (sedia)

Hammock (amaca), swan (cigno), scoop (paletta), tractor (trattore),

\section{APPENDIX B}

Materials used in Experiments 2 and 3 organized by Semantic Context (within-category close, within-category far, and unrelated). Within-category close blocks were determined by each row (e.g. dog, wolf, fox). Within-category far related blocks were designed using a "column" restricted to each set (e.g. dog, mouse, lizard). Unrelated blocks contained the homologous object in each set restricted by group (e.g. dog, fork, orange). The shape pictures used in Experiment 3 (unrelated fillers) were circle, triangle, diamond.

\section{Group 1}

Animals: $\quad$ dog, wolf, fox mouse, squirrel, chipmunk

lizard, frog, snake

Tools: $\quad$ fork, spoon, ladle plate, cup, bowl knife, razor, scissors

Fruits/vegetables: orange, lime, lemon broccoli, cauliflower, lettuce cucumber, asparagus, celery

\section{Group 2}

Animals: horse, donkey, zebra ant, mosquito, bee eagle, parrot, owl

Tools: $\quad$ wrench, screwdriver, pliers shovel, rake, hoe bolt, nail, screw

Vehicles: truck, van, bus bicycle, scooter, motorcycle wagon, carriage, sleigh 